\title{
October 2014 Critical Care Case of the Month: A Skin Rash in the ICU
}

\author{
Robert A. Raschke, MD \\ Banner Good Samaritan Medical Center \\ Phoenix, AZ
}

\section{History of Present IIIness}

A 28 year old man was admitted to an outside hospital with an ethylene glycol overdose in an apparent suicide attempt. At that time the patient was delirious and vomiting. He had a severe metabolic acidosis and a creatinine of $2.1 \mathrm{mg} / \mathrm{dL}$. He was intubated, a nasogastric tube was placed, and he was transferred to the toxicology service.

\section{$\mathrm{PMH}, \mathrm{FH}, \mathrm{SH}$}

There was no significant past medical history. Family history was noncontributory. He was a nonsmoker who recently had a fight with his girlfriend prompting the suicide attempt.

\section{Physical Examination}

Vital signs were stable and he was sedated and nasally intubated receiving mechanical ventilation. There were no other significant findings on physical examination

Which of the following can be used for ethylene glycol poisoning?
1. Ethanol
2. Fomezipole
3. Hemodialysis
4. Pyridoxine
5. All of the above 


\section{Correct! \\ 5. All of the above.}

Fomepizole or 4-methylpyrazole is indicated for use as an antidote in confirmed or suspected ethylene glycol or methanol poisoning (1). It may be used alone or in combination with hemodialysis. Fomepizole is a competitive inhibitor of the enzyme alcohol dehydrogenase found in the liver. This enzyme plays a key role in the metabolism of ethylene glycol and methanol. Ethylene glycol is first metabolized to glycolaldehyde by the enzyme alcohol dehydrogenase, which then undergoes further oxidation to glycolate, glyoxylate, and oxalate. It is glycolate and oxalate that are primarily responsible for the metabolic acidosis and renal damage that are seen in ethylene glycol poisoning. By competitively inhibiting the first enzyme in the metabolism of ethylene glycol fomepizole slows the production of the toxic metabolites.

In patients with ethylene glycol poisoning, the administration of pyridoxine and has been frequently advocated as an adjunctive therapy, because pyridoxine is a cofactor in the metabolism of glycolic acid to glycine. However, there are sparse data supporting any beneficial effect of such treatment.

The patient was treated with fomepizole, hemodialysis, pyridoxine, folate and thiamine and improved. He was extubated 24 hours after transfer and was awake and alert.

However, 30 hours after transfer he developed tachycardia to 150 beats/min, fever to $103^{\circ} \mathrm{F}$ and hypotension. His chest $\mathrm{x}$-ray and echocardiogram were negative.

Which of the following should be administered next?

1. Antibiotics

2. Fomepizole and pyridoxine

3. Intravenous fluids

4. 1 and 3

5. All of the above 


\section{Correct!}

\section{1 and 3}

The patient is clinically presenting with a sepsis syndrome treated with antibiotics and fluids. Ethylene glycol poisoning does not present with "relapses".

On physical examination he had a heart rate of 150 beats/min, a blood pressure of $85 / 62 \mathrm{~mm} \mathrm{Hg}$, a respiratory rate of 28 and a temperature $102.8^{\circ} \mathrm{F}$. He was confused and agitated, he had an exudate coming from his right nares and blood from his left nares. In addition, he developed an erythroderma on his hands and feet (Figure 1).

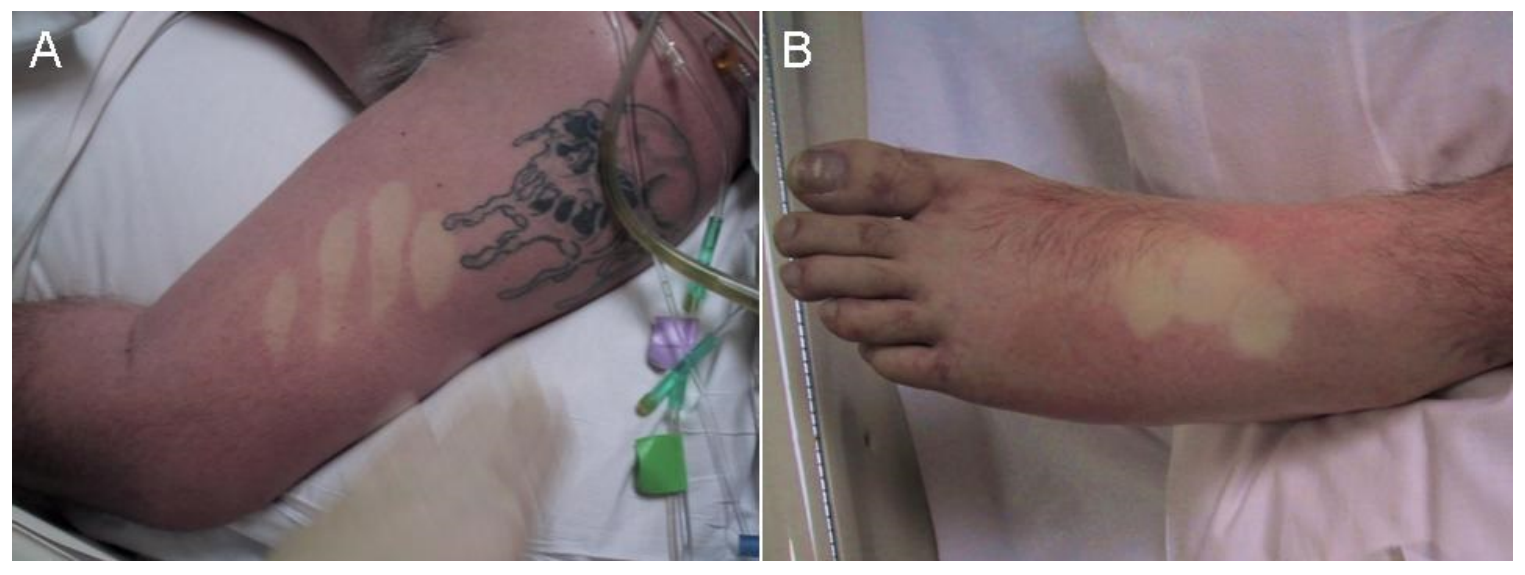

Figure 1. Photographs of arm and foot showing erythroderma.

Which of the following statements is false?

1. He likely has a drug reaction and his antibiotics should be stopped

2. He may have lactic acidosis

3. His blood cultures may be negative

4. Nares culture will likely show Staphylococcus aureus

5. Radiography may show sinusitis 


\section{Correct! \\ 1. He likely has a drug reaction and his antibiotics should be stopped}

The physical findings of a discharge and blood coming from his nares suggest a possible sinusitis. This was confirmed by CT scan (Figure 2).

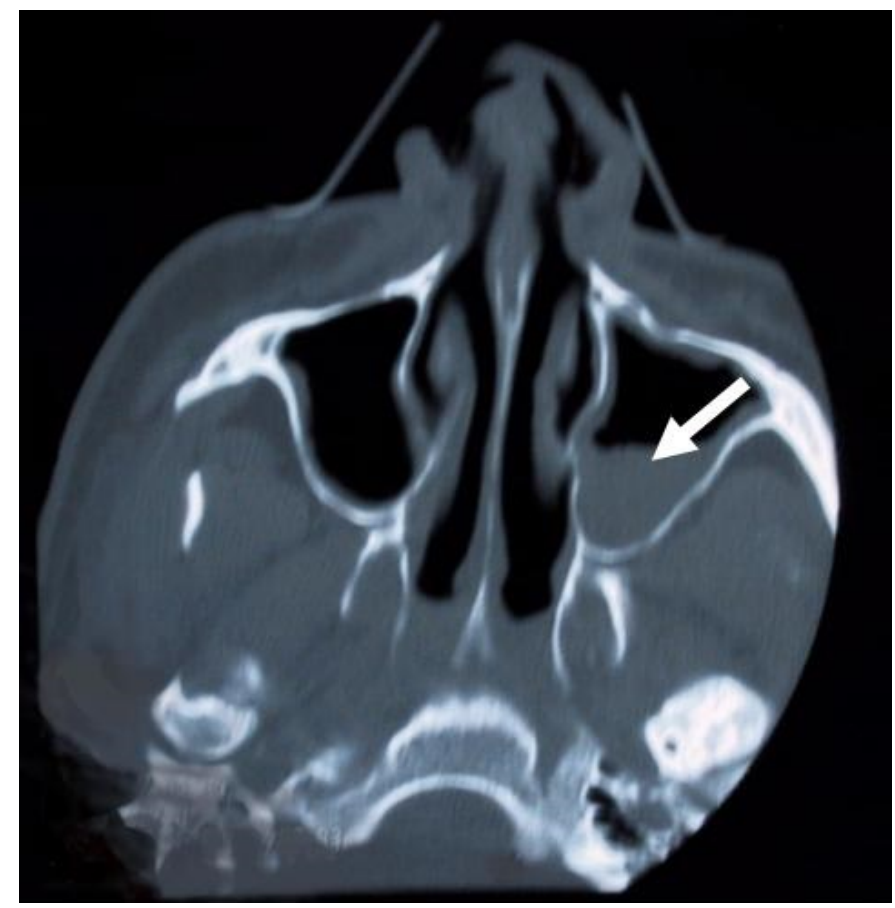

Figure 2. CT scan of sinuses showing an air-fluid level in the left maxillary sinus (arrow).

The erythroderma is a second clue on physical examination. In this setting it suggests possible toxic shock syndrome secondary to Staphylococcal or Streptococcal disease. Toxic shock syndrome results when bacteria release toxins which result in an acute multi-system disease often accompanied by multisystem organ failure (2). Major criteria for toxic shock syndrome are listed in table 1.

Table 1. Criteria for toxic shock syndrome.

- Fever $>102^{\circ} \mathrm{F}$.

- Hypotension

- Erythroderma

- Desquamation (1-3 weeks later)

- >3 organs involved (Gl-diarrhea, muscles, mucous membranes, renal, hepatic enzymes, thrombocytopenia, CNS effects)

- Blood cultures negative (may be positive with Staphylococcal disease) 
Which of the following statements are $\underline{\text { true }}$ regarding toxic shock syndrome?

1. Clindamycin can suppress Staphylococcal toxin production

2. High dose intravenous immunoglobin (IVIG) has been shown to not be beneficial

3. The vast majority of cases are menstrually associated

4. Treatment with antibiotics is without benefit

5. All of the above 


\section{Correct! \\ 1. Clindamycin can suppress Staphylococcal toxin production}

Toxic shock syndrome results from the ability of bacterial toxins, usually TSST-1 in Staphylococcal disease, to act as superantigens, stimulating immune-cell expansion and rampant cytokine expression (2). A repetitive cycle of cell stimulation and cytokine release results in a cytokine avalanche that causes tissue damage, disseminated intravascular coagulation, and organ dysfunction. About $50 \%$ of cases are menstrual and $50 \%$ non-menstrual from surgical site infections, sinusitis, post-influenza pneumonia, etc. Therapy consists of administration on antimicrobial agents including drugs capable of suppressing toxin production (e.g., clindamycin, linezolid). Intravenous immunoglobulin has the potential to neutralize superantigen and to mitigate subsequent tissue damage.

Our patient did recover with administration of nafcillin, vancomycin, clindamycin and IVIG. However, he did develop skin desquamation about 2 weeks after his initial event (Figure 3).

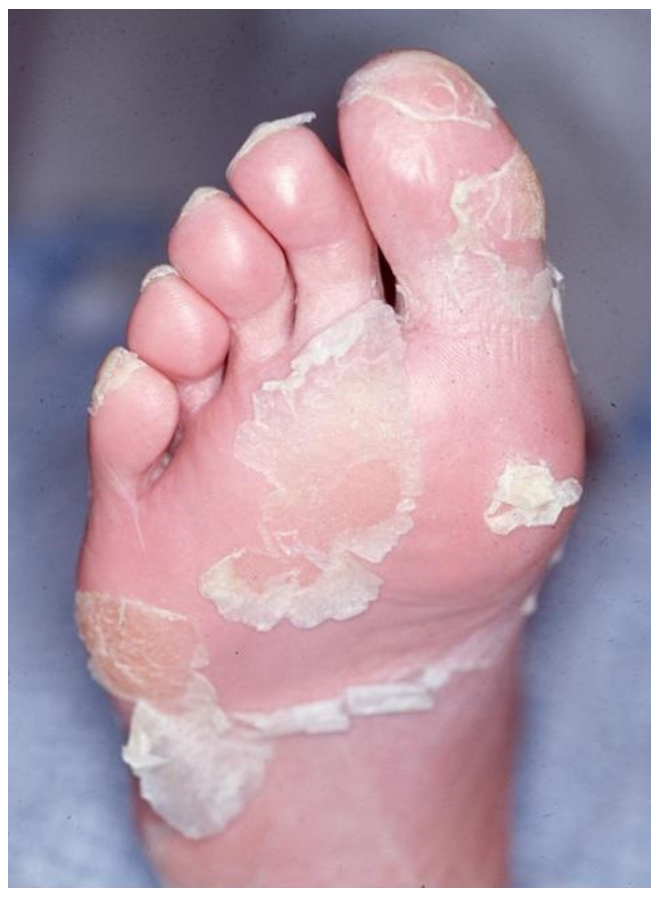

Figure 3. Photography of patient's foot showing skin desquamation.

\section{References}

1. Brent J. Fomepizole for ethylene glycol and methanol poisoning. N Engl J Med. 2009;360(21):2216-23. [CrossRef] [PubMed]

2. Lappin E, Ferguson AJ. Gram-positive toxic shock syndromes. Lancet Infect Dis. 2009 May;9(5):281-90. [CrossRef] [PubMed] 